\title{
Novel Techniques for Imaging the Heart: Cardiac MR and CT
}

M. Di Carli and R. Kwong, Eds.

Oxford, U.K.: Wiley-Blackwell, 2008, 360 pages, $\$ 124.95$

This book addresses the gap in the knowledge base and technology that exists between cardiology and radiology. The most current information is provided on cardiovascular disease and on applying CT and MRI in managing these types of patients. The intended audience is cardiology, radiology, and nuclear medicine physicians, although cardiovascular surgeons and their trainees (residents and fellows) in all these disciplines can benefit from the information. Specialty nurses and physician assistants can also use the information.

The book is divided into 3 parts.

Part I involves the general considerations and fundamentals of imaging. The 4 chapters in this part address the general principles of CT and MRI, including the contrast agents used in these imaging modalities. Radiation dose and safety considerations are also discussed in this part.

The next 12 chapters constitute Part II and describe clinical applications of CT and MRI. The topics include the evaluation of symptomatic patients with suspected coronary artery disease, chest pain in patients with known coronary artery disease, patients with left ventricular dysfunction for potential revascularization, patients with new-onset heart failure, patients before noncardiac surgery, patients before interventional electrophysiology, and blood flow and heart valve disease. Other chapters discuss CT and MRI evaluation of atherosclerotic plaque, hybrid imaging (PET/CT and SPECT/CT), and the merits of CT angiography and MR angiography for coronary artery imaging. One chapter is dedicated to a critical review of imaging approaches for the diagnosis and prognosis of coronary artery disease, and one other chapter addresses the improvement of patient management by using atherosclerosis imaging.
Part III consists of 7 chapters dedicated to advanced applications of CT and MRI. Included are biologic and clinical perspectives on atherosclerosis, stem cell imaging, imaging of myocardial ischemia and viability, myocardial mechanics, cardiovascular interventional MRI, high-field-strength MRI, and multidetector CT for coronary artery stenoses and physiology.

This book has multiple coauthors from multiple disciplines. Each chapter has an introduction that presents the topics to be discussed, and the format is consistently followed. The material is easy to read, the images of excellent quality, and the legends clear and precise. Thorough reference lists are given at the end of each chapter. It is difficult to select an outstanding chapter given that all are excellent and present a systematic, practical, and in-depth approach to imaging. A great plus to this book is the enclosed CD-ROM, which has video clips and a database of figures from the book. The video clips are discussed in the chapters and easily selected using the icon.

This is an excellent, highly recommended book that belongs in the libraries of physicians, trainees, and allied health professionals who deal with patients having cardiovascular disease. The authors are commended for adding this volume to the medical literature. I would suggest that for the next edition they consider including information on cancer patients and chemotherapy.

Aurelio Matamoros, Jr. M.D. Anderson Cancer Center Houston, Texas 